\title{
Stratosphere-Troposphere Coupling in a Relatively Simple AGCM: The Role of Eddies
}

\author{
PAUl J. Kushner \\ NOAA/Geophysical Fluid Dynamics Laboratory, Princeton, New Jersey \\ LORENZO M. POLVANI* \\ Department of Applied Physics and Applied Mathematics and the Department of Earth and Environmental Sciences, Columbia University, \\ New York, New York
}

(Manuscript received 16 January 2003, in final form 29 July 2003)

\begin{abstract}
The extratropical circulation response to cooling of the polar-winter stratosphere in a simple AGCM is investigated. The AGCM is a dry hydrostatic primitive equation model with zonally symmetric boundary conditions and analytically specified physics. It is found that, as the polar-winter stratosphere is cooled, the tropospheric jet shifts poleward. This response projects almost entirely and positively (by convention) onto the AGCM's annular mode. At the same time, the vertical flux of wave activity from the troposphere to the stratosphere is reduced and the meridional flux of wave activity from high to low latitudes is increased. Thus, as the stratosphere is cooled, the stratospheric wave drag is reduced.

In order to understand this response, the transient adjustment of the stratosphere-troposphere system is investigated using an ensemble of "switch on" stratospheric cooling runs of the AGCM. The response to the switch-on stratospheric cooling descends from the upper stratosphere into the troposphere on a time scale that matches simple downward-control theory estimates.

The downward-control analysis is pursued with a zonally symmetric model that uses as input the thermal and eddy-driving terms from the eddying AGCM. With this model, the contributions to the response from the thermal and eddy-driving perturbations can be investigated separately, in the absence of eddy feedbacks. It is found that the stratospheric thermal perturbation, in the absence of such feedbacks, induces a response that is confined to the stratosphere. The stratospheric eddy-driving perturbation, on the other hand, induces a response that penetrates into the midtroposphere but does not account, in the zonally symmetric model, for the tropospheric jet shift. Furthermore, the tropospheric eddy-driving perturbation, in the zonally symmetric model, induces a strong upward response in the stratospheric winds. From these experiments and from additional experiments with the eddying AGCM, it is concluded that the stratospheric eddy-driving response induces a tropospheric response, but that the full circulation response results from a two-way coupling between the stratosphere and the troposphere.
\end{abstract}

\section{Introduction}

There is growing interest in the possibility that the extratropical stratosphere and troposphere might strongly influence each other. On time scales of years to decades, it is well known that the stratospheric and tropospheric geopotential fields are well correlated (e.g., Baldwin et al. 1994; Perlwitz and Graf 1995). More recent work has uncovered the existence of vertically deep annular modes that extend from the troposphere into the stratosphere. The annular modes have circula-

* Additional affiliation: Visiting Scientist in the Atmospheric and Oceanic Sciences Program, Princeton University, Princeton, New Jersey.

Corresponding author address: Dr. Paul J. Kushner, NOAA/GFDL, P.O. Box 308, Forrestal Campus, Princeton, NJ 08542.

E-mail: paul.kushner@noaa.gov tion and surface-temperature spatial patterns that correlate well with extratropical trends in both hemispheres that have been observed since the 1960s (Thompson and Wallace 1998, 2000; Thompson et al. 2000; Thompson and Solomon 2002). The cited studies, most convincingly the Thompson and Solomon study, suggest that at least some of these trends are stratospherically forced, implying that stratosphere-troposphere interaction may be important in the climate system.

On time scales of weeks to months, anomalously strong or weak stratospheric polar-vortex events have been shown to set off annular-mode signals that propagate downward from the stratosphere to the troposphere (Baldwin and Dunkerton 1999, 2001). These signals can be linked to anomalies in the sea level pressure, surface temperatures, and storm tracks as much as 60 days after the initial stratospheric signal (Baldwin and Dunkerton 2001). Taken at their face value, these results suggests that the stratosphere-troposphere interaction 
may exert a significant control on tropospheric weather and weather predictability. But the dynamical mechanisms for such control remain unclear and controversial (e.g., Plumb and Semeniuk 2003).

There is also controversy on this issue in the general circulation model literature, starting with the studies of Shindell et al. (1999, 2001), who show that the only way that recent annular mode trends can be realistically simulated in their particular coupled GCM is by including an enhanced stratosphere. This result, however, does not appear to be robust. In particular, two studies (Fyfe et al. 1999; Gillett et al. 2002) have shown that positive annular mode responses can be forced by greenhouse warming in other GCMs, independently of having a highly resolved stratosphere.

This controversy and lack of robustness has prompted us (Polvani and Kushner 2002, hereafter PK) to set up a very simple GCM that is able to simulate the main elements of the extratropical stratosphere-troposphere system, and then to cool the model's stratosphere to see whether the tropospheric circulation can be changed in any significant way. Our aims in this pursuit are to create a model that other investigators can easily reproduce, to be able to demonstrate that our results are robust, and, ultimately, to explain the dynamics of any stratospheric influence on the troposphere seen in such a model.

Using a simple model, similar to those used by Scinocca and Haynes (1998) and Taguchi et al. (2001) and briefly reviewed in section 2 , we (PK) have found a novel and robust result that demonstrates one way in which the stratosphere might influence the tropospheric circulation. From a sequence of experiments in which the winter polar stratosphere is successively cooled from a reference value, we have found that, for sufficiently strong stratospheric cooling, a remarkable poleward shift of the tropospheric circulation occurs. The tropospheric response projects almost completely and positively onto the model's annular mode. We also find that the response is insensitive to vertical and horizontal resolution beyond a minimum threshold. This result lends support, within a relatively simple model, to the idea that stratospheric diabatic heating perturbations can induce strong tropospheric responses (Shindell et al. 1999, 2001; Thompson and Solomon 2002).

In PK then, we have been able to create a reproducible and robust example of stratosphere-troposphere coupling. The aim of this study is, now, to explain the dynamics of this coupling. In section 2 , we review the model. In section 3, we describe the characteristics of the response of the mean flow and of the eddy driving to stratospheric thermal perturbations. In section 4, we examine the transient adjustment of the stratospheretroposphere system to the stratospheric cooling perturbation using an ensemble of transient switch-on integrations. In section 5, we pursue a "downward control" analysis in which we analyze the response using a zonally symmetric model and additional integrations of the eddying model. We provide a brief conclusion in section 6 . The appendix outlines the setup of the zonally symmetric model.

\section{Description and characteristics of the model}

Our model is a dry, hydrostatic, primitive equations model, with parameter settings, forcing, and dissipation that are similar to those in the "Held-Suarez benchmark" (Held and Suarez 1994, hereafter HS). It uses the spectral transforms method in the horizontal and Simmons and Burridge (1981) finite differencing in the vertical. Diabatic heating is represented by Newtonian relaxation of the temperature toward a prescribed, zonally symmetric, equilibrium temperature field. Planetary boundary layer drag is represented by Rayleigh damping. The model has a flat lower boundary; since the diabatic heating is also zonally symmetric, the model is statistically zonally homogeneous and has no stationary planetary-scale eddies. Details about the numerics can be found in PK.

Unlike the HS benchmark, whose stratospheric equilibrium temperature profile is isothermal, our model's stratospheric equilibrium temperature profile roughly represents the observed solsticial stratospheric radiativeconvective equilibrium temperature profile as shown, for example, in Fig. 1.2 of Andrews et al. (1987). The equilibrium temperature, $T_{\text {eq }}$, is defined as follows:

$$
T_{\text {eq }}(\phi, p)= \begin{cases}T_{\text {eq }}^{\text {strat }}(p, \phi), & p<p_{T} \\ T_{\text {eq }}^{\text {trop }}(p, \phi), & p \geq p_{T},\end{cases}
$$

where $p$ is pressure, $\phi$ is latitude, and $p_{T}=100 \mathrm{mb}$ is a nominal tropopause height. The tropospheric relaxation temperature, $T_{\text {eq }}^{\text {trop }}$, is nearly identical to that of $\mathrm{HS}$ and is given in PK. The stratospheric relaxation temperature is given by

$$
\begin{aligned}
T_{\mathrm{eq}}^{\mathrm{strat}}(p, \phi)= & {[1-W(\phi)] T_{\mathrm{US}}(p) } \\
& +W(\phi) T_{\mathrm{PV}}(p ; \gamma),
\end{aligned}
$$

where $T_{\mathrm{US}}(p)$ is from the U.S. Standard Atmosphere 1976 [United States Committee on Extension to the Standard Atmosphere (COESA), 1976],

$$
T_{\mathrm{PV}}(p ; \gamma)=T_{\mathrm{US}}\left(p_{T}\right)\left(p / p_{T}\right)^{R \gamma / g}
$$

is the temperature of a hydrostatic atmosphere with thermal lapse rate $\gamma$, and $W(\phi)$ is a weight function used to confine the cooling over the winter pole,

$$
W(\phi)=\frac{1}{2}\left\{1-\tanh \left[\frac{\left(\phi-\phi_{0}\right)}{\Delta \phi}\right]\right\},
$$

with $\phi_{0}=-50^{\circ}$ latitude (i.e., $50^{\circ} \mathrm{S}$ latitude) and $\Delta \phi=$ $10^{\circ}$ latitude. The stratospheric equilibrium temperature makes a smooth transition in latitude from the U.S. Standard Atmosphere in the summer (northern) hemisphere to a "cold-polar vortex" profile in the winter (southern) hemisphere extratropics. 
Since $T_{\text {eq }}$ is time invariant, the model has no seasonal cycle. This, together with the fact that the model has no stationary planetary eddies, makes the model a simpler starting point for analyzing stratosphere-troposphere interactions than the dry AGCM simulations of Taguchi and Yoden (2002a,b), which include topographic forcing in addition to baroclinic-eddy forcing, and which include a stratospheric seasonal cycle.

The winter-hemisphere lapse rate, $\gamma$, is the parameter that we vary to impose the thermal perturbation in the stratosphere. The equilibrium temperature, $T_{\text {eq }}$, for the cases where $\gamma=2 \mathrm{~K} \mathrm{~km}^{-1}$ and $\gamma=4 \mathrm{~K} \mathrm{~km}^{-1}$ in (3), are shown in Figs. 1a,b. By design, the value of $\gamma$ determines the strength of the equator-to-winter-pole equilibrium temperature gradient and, as a result, the strength of the spunup polar vortex in the model. These two cases are the focus of this study. The difference in equilibrium temperature between the $\gamma=2 \mathrm{~K} \mathrm{~km}^{-1}$ and $\gamma=4 \mathrm{~K} \mathrm{~km}^{-1}$ cases is shown in Fig. 1c.

The resolution of the integrations analyzed here is T42 horizontal resolution and 40-level vertical resolution. As shown in PK, all key results are largely insensitive to resolution, beyond a minimum threshold. We note that a sponge that damps the winds toward zero occupies the top six layers of the 40-level model, starting at $0.5 \mathrm{mb}$, just above the nominal summer stratopause seen in the equilibrium-temperature profile in Fig. 1. Thus, for all subsequent figures, fields are plotted below $1 \mathrm{mb}$ only. Another point regarding the sponge is worth mentioning: we have repeated our standard calculations with the value of sponge damping reduced by a factor of 2 over the standard PK case, and have found that our results in the lower stratosphere and troposphere are insensitive to this change.

\section{Equilibrium response to stratospheric cooling}

The key result of PK, whose dynamics we wish to explore in this study, is that although the equilibrium temperature difference between the $\gamma=2$ and $\gamma=4$ cases, illustrated in Fig. 1c, is confined to the winterhemisphere stratosphere, the mean circulation responds significantly in the troposphere. This is illustrated in Figs. 2a,b, which shows the zonal- and time-mean zonal winds for $\gamma=2 \mathrm{~K} \mathrm{~km}^{-1}$ and $\gamma=4 \mathrm{~K} \mathrm{~km}^{-1}$, respectively. The time means are averages over the last 9000 days of a 10 000-day integration. Figure 2c shows the difference between the two cases. In the figure, only the winter (southern) hemisphere is shown, since the summer hemisphere does not respond significantly. Not surprisingly, the stratospheric polar vortex strengthens and broadens as $\gamma$ goes from 2 to $4 \mathrm{~K} \mathrm{~km}^{-1}$. The polarvortex strengthening is expected because $\left|\partial\left(T_{\text {eq }}^{\text {strat }}\right) / \partial \phi\right|$ increases and the polar-vortex broadening is expected because the region of nonzero gradients of $T_{\mathrm{eq}}^{\text {strat }}$ expands equatorward (not shown).

More surprising is the change in the tropospheric circulation, where the main jet shifts poleward by $10^{\circ}-15^{\circ}$ latitude (PK). (For clarity, the $30^{\circ} \mathrm{S}$ latitude is marked by a vertical line in Figs. $2 \mathrm{a}$ and $2 \mathrm{~b}$ ). This response is strong even at the surface (PK, Fig. 2) and projects strongly onto the model's annular mode pattern (PK, Fig. 4), which also represents the meridional migration of the tropospheric jet. We recall, finally, that this response is found to be largely independent of the model's horizontal and vertical resolution (PK, Fig. 3).

As a first step toward a dynamical understanding of this response, we perform standard Eliassen-Palm (EP) flux diagnostics. These reveal that accompanying the changes to the mean circulation are substantial changes to the eddy driving of the mean flow. Following Fusco and Salby (1999), Newman et al. (2001), and $\mathrm{Hu}$ and Tung (2002), we calculate an EP flux budget over selected stratospheric regions. We use the hydrostatic primitive equation zonal-mean EP flux in pressure coordinates, which is defined, using pressure as the vertical coordinate, and standard notation, by

$$
\begin{aligned}
\mathbf{F} & =\left[F_{(\phi)}, F_{(p)}\right], \\
F_{(\phi)} & =a \cos \phi\left[-\overline{u^{\prime} v^{\prime}}+\left(\frac{\partial \bar{u}}{\partial p}\right) \overline{v^{\prime} \theta^{\prime}} /\left(\frac{\partial \bar{\theta}}{\partial p}\right)\right], \\
F_{(p)} & =a \cos \phi\left[(f+\bar{\zeta}) \overline{v^{\prime} \theta^{\prime}} /\left(\frac{\partial \bar{\theta}}{\partial p}\right)-\overline{u^{\prime} \omega^{\prime}}\right] .
\end{aligned}
$$

The zonal-mean angular momentum equation can be written

$$
\frac{\partial M}{\partial t}=\cdots+\boldsymbol{\nabla} \cdot \mathbf{F}
$$

where $M=a \cos \phi \bar{u}$ is the zonal-mean angular momentum per unit mass and the ellipsis represents the residual circulation and dissipation terms. For the budget, we use the divergence theorem to break up the integral of $\boldsymbol{\nabla} \cdot \mathbf{F}$ into four terms. These terms represent the flux through each side of a rectangle defined by the intervals $p_{1} \leq p \leq p_{2}$ and $\phi_{1} \leq \phi \leq \phi_{2}$ in the $\phi$ - $p$ plane:

$$
\begin{aligned}
\int_{p_{1}}^{p_{2}} \int_{\phi_{1}}^{\phi_{2}} \nabla \cdot \mathbf{F} \cos \phi d \phi d p \\
=\left.a^{-1} \cos \phi \int_{p_{1}}^{p_{2}} F_{(\phi)} d p\right|_{\phi_{2}}-\left.a^{-1} \cos \phi \int_{p_{1}}^{p_{2}} F_{(\phi)} d p\right|_{\phi_{1}} \\
\quad+\left.\int_{\phi_{1}}^{\phi_{2}} \cos \phi F_{(p)} d \phi\right|_{p_{2}}-\left.\int_{\phi_{1}}^{\phi_{2}} \cos \phi F_{(p)} d \phi\right|_{p_{1}}
\end{aligned}
$$

The integral and the four terms have dimensions of mass-length per time to the fourth power.

We construct this budget for two boxes: a "high latitude" box that extends from $\phi_{1}=90^{\circ} \mathrm{S}$ to $\phi_{2}=40^{\circ} \mathrm{S}$ and a "low latitude" box that extends from $\phi_{1}=40^{\circ} \mathrm{S}$ to $\phi_{2}=21^{\circ} \mathrm{S}$. Both boxes are bounded in pressure at 

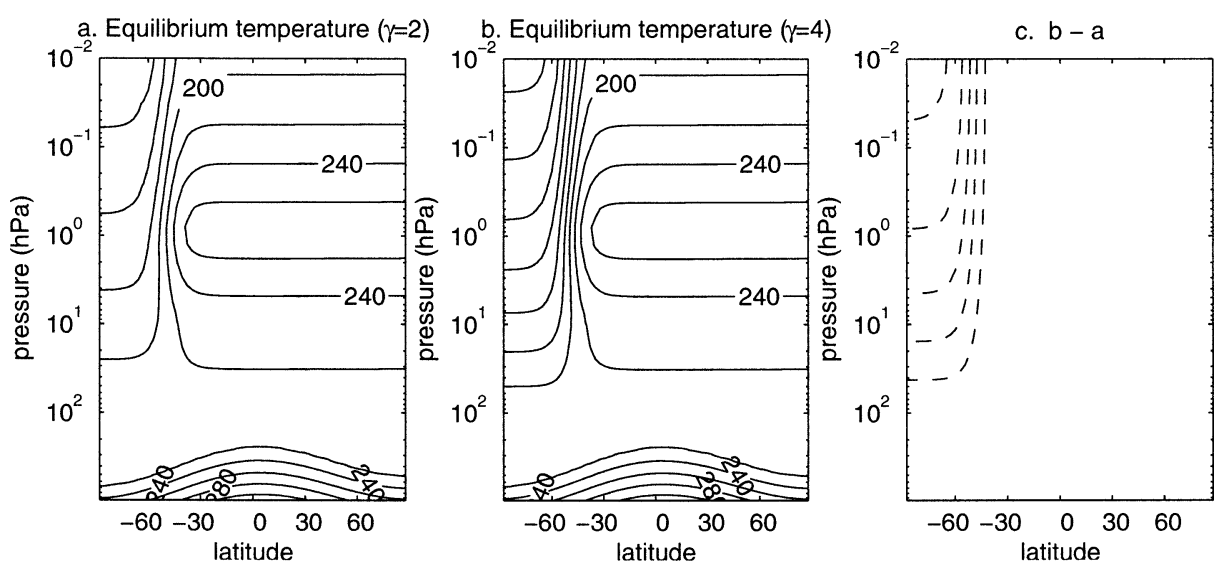

FIG. 1. (a) Plot of $T_{\mathrm{eq}}$ for $\gamma=2 \mathrm{~K} \mathrm{~km}^{-1}$. The contour interval is $20 \mathrm{~K}$. (b) As in (a) but for $\gamma=4$ $\mathrm{K} \mathrm{km}^{-1}$. (c) The difference in $T_{\mathrm{eq}}$ between $\gamma=4 \mathrm{~K} \mathrm{~km}^{-1}$ and $\gamma=2 \mathrm{~K} \mathrm{~km}^{-1}$, that is, (b) minus (a). The contour interval is $10 \mathrm{~K}$, dashed contours denote negative values, and the zero contour is not plotted.

$p_{1}=97 \mathrm{mb}$ and $p_{2}=1 \mathrm{mb} \cdot{ }^{1}$ The boundary of the highlatitude box is chosen to capture the changes to the wave activity in the vicinity of the polar vortex, and the lowlatitude box to capture changes in the vicinity of the tropospheric jet. Figure 3 depicts, for $\gamma=2$ (red) and $\gamma=4$ (blue), the budget for the high- and low-latitude boxes (see caption for details).

In the high-latitude box, we see a substantial reduction-using the units of the figure, $(3.6-6.0) / 6.0=$ $-40 \%$-in the amount of wave activity absorbed, from $\gamma=2$ to $\gamma=4$. This reduction arises from a modest decrease in vertical flux from the troposphere [(8.9 $10.2) / 10.2=-13 \%$ ] into the stratosphere and a substantial increase of meridional flux out of the high-latitude box and into the low-latitude box $[(4.6-3.5) / 3.5$ $=+30 \%$ ].

\footnotetext{
${ }^{1}$ The pressure interpolation levels were chosen to correspond close-
} ly to the model's sigma-coordinate surfaces.
In the low-latitude box, the absorbed wave activity also decreases, by $(9.3-6.5) / 9.3=30 \%$ from $\gamma=2$ to $\gamma=4$, despite the increased meridional flux into this box from higher latitudes. The main cause for this decrease in absorbed wave activity at lower latitudes is a dramatic reduction $[(3.7-8.5) / 8.5=-56 \%]$ in the vertical flux from the troposphere into this box.

The large reduction in vertical flux from the troposphere to the stratosphere at low latitudes box is illustrated in Fig. 4, which shows the vertical component of the EP flux across the $97-\mathrm{mb}$ pressure surface, as a function of zonal wavenumber and latitude, for $\gamma=2$ and for $\gamma=4$. This figure represents the latitude/Fourier-wavenumber decomposition of the arrows pointing up into the bottom of the boxes in Fig. 3. The most pronounced change in the upward flux is at the synoptic wave scale (wavenumber 5-7): for $\gamma=2$ (Fig. 4a) there is an EP flux source at this scale that is greatly reduced for $\gamma=4$ (Fig. 4b).
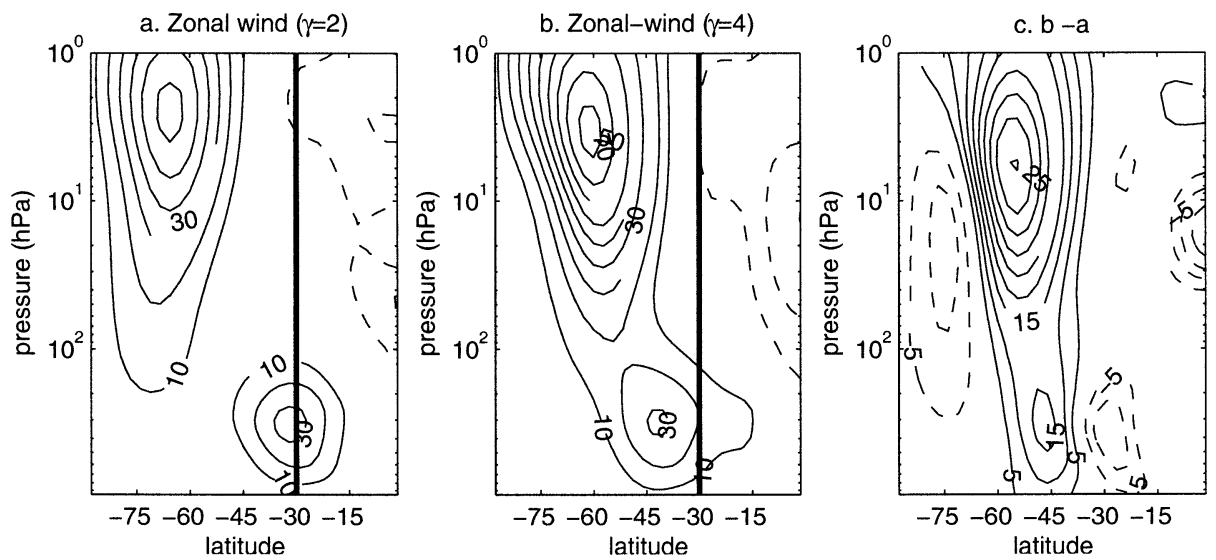

FIG. 2. (a) The time- and zonal-mean zonal winds for $\gamma=2 \mathrm{~K} \mathrm{~km}^{-1}$. The contour interval is $10 \mathrm{~m}$ $\mathrm{s}^{-1}$ and the zero contour is not plotted. The latitude $30^{\circ} \mathrm{S}$ is marked with a heavy vertical line. (b) As in (a) but for $\gamma=4 \mathrm{~K} \mathrm{~km}^{-1}$. (c) The difference in the time- and zonal-mean zonal wind between $\gamma=$ $4 \mathrm{~K} \mathrm{~km}^{-1}$ and $\gamma=2 \mathrm{~K} \mathrm{~km}^{-1}$, that is, (b) minus (a). The contour interval is $5 \mathrm{~m} \mathrm{~s}^{-1}$ and the zero contour is not plotted. 


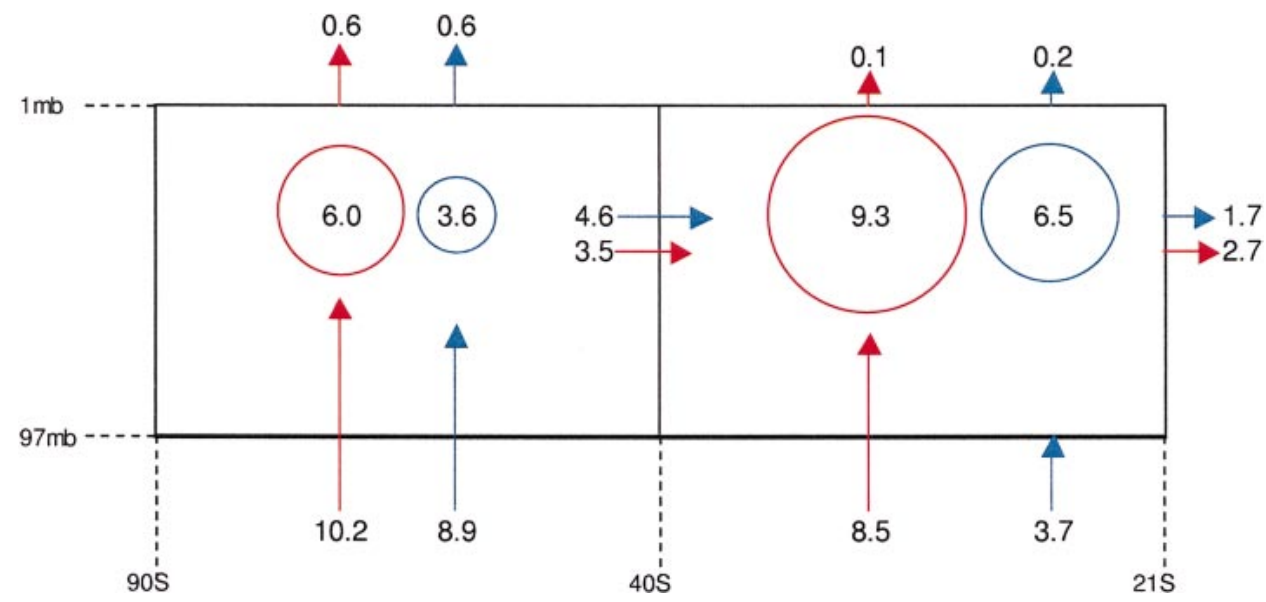

FIG. 3. The EP flux budget for $\gamma=2$ (red) and for $\gamma=4$ (blue). Numbers are in units of $10^{4} \mathrm{~kg} \mathrm{~m} \mathrm{~s}^{-4}$. Arrows pointing into the bottom of the boxes denote the upward EP flux from below $97 \mathrm{mb}$; arrows pointing out the top, the upward flux from below $1 \mathrm{mb}$; arrows pointing out the right at $40^{\circ} \mathrm{S}$, the meridional flux from the high-latitude into the low-latitude box; and arrows pointing out the right at $21^{\circ} \mathrm{S}$, the meridional flux out of the low-latitude box. The numbers in the center of the circles denote the integrated EP flux convergence within the box. The integrated EP flux convergence is positive in all regions, indicating, as expected, that there is a net convergence of EP wave activity, that is, a net eddy-driven drag. The arrows and circles are approximately scaled to provide an idea of the relative size of the contributions.

To summarize, we have found that as the polar winter stratosphere is cooled,

- the stratospheric polar vortex strengthens and broadens,

- the tropospheric jet shifts poleward and this response projects strongly onto the model's annular mode,

- the planetary wave drag in the stratosphere is reduced,

- wave activity is deflected more from high latitudes to low latitudes, and

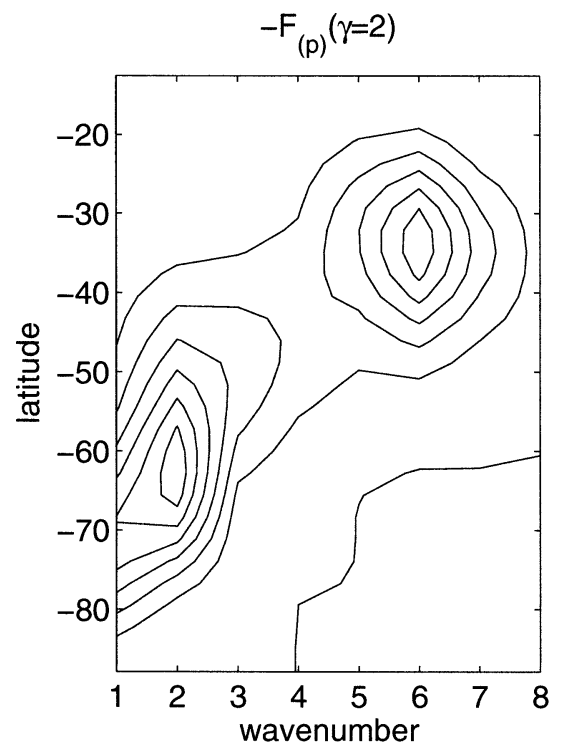

- the upward flux of synoptic-scale wave activity into the stratosphere is greatly reduced.

However useful, the EP flux analysis does not, unfortunately, answer the important question of cause and effect. For example, does the strengthening of the polar vortex reduce the eddy driving or does the reduction in eddy driving strengthen the polar vortex? Or, what causes the change to the eddy driving at the synoptic scale? Such questions are difficult to answer through the di-

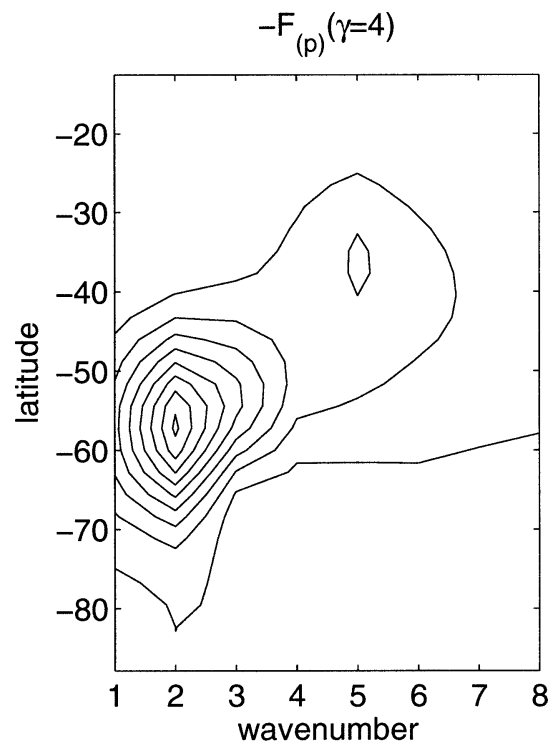

FIG. 4. Plot of $-F_{(p)}$ at $97 \mathrm{mb}$ for (a) $\gamma=2$ and (b) $\gamma=4$. The minus sign is included so that positive contours correspond to an upward flux. The contour interval is $2.5 \times 10^{4}$ $\mathrm{kg} \mathrm{m} \mathrm{s}{ }^{-4}$. 

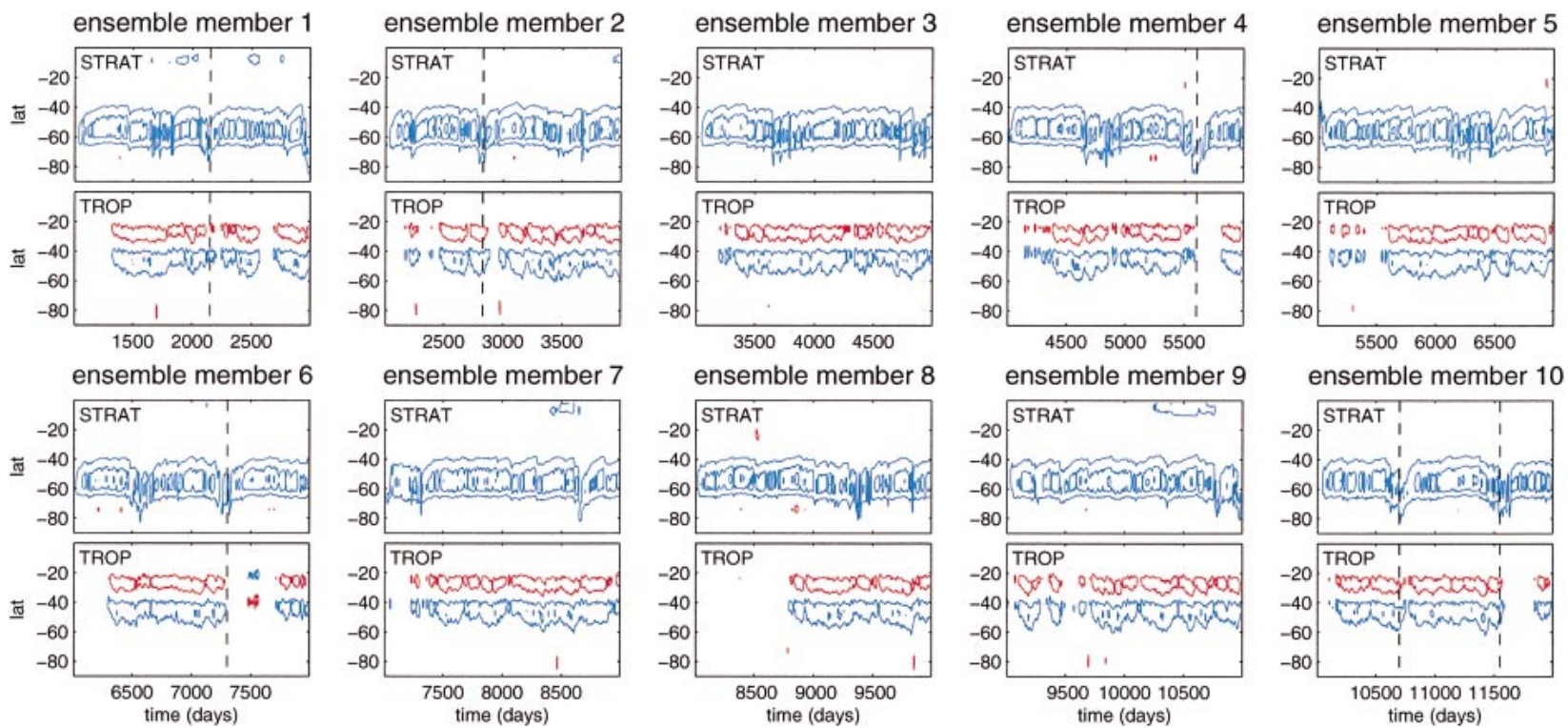

FIG. 5. Departure, in zonal-mean zonal wind, of individual ensemble members from the long-term mean of $\gamma=2$, at 6 mb (labeled STRAT) and $520 \mathrm{mb}$ (labeled TROP) model levels. The contour interval in the STRAT panels is $20 \mathrm{~m} \mathrm{~s}^{-1}$; in the TROP panels, $10 \mathrm{~m} \mathrm{~s}$. The zero contour is not plotted. Blue contours indicate positive values and red contours indicate negative values. Vertical dashed lines indicate selected events where a reduction in the stratospheric response is followed by a reduction in the tropospheric response.

agnosis of the equilibrated eddy and mean response. As an alternative, in the next section, we analyze the transient response to stratospheric thermal perturbations with the idea that directly tracking the adjustment of the system to the perturbation will provide insight into the equilibrium response's dynamics.

\section{Transient response to stratospheric cooling}

Starting from an equilibrated $\gamma=2$ integration, we perform a set of "switch-on forcing" integrations, in which the value of $\gamma$ is changed from 2 to $4 \mathrm{~K} \mathrm{~km}^{-1}$ instantaneously. We first extend the original $\gamma=2$ integration from 10000 to 12000 days and then perform an ensemble of ten 2000-day switch-on forcing integrations starting from day 1001, 2001, ., 10001 of the $\gamma=2$ integration. We choose the thousand-day separation interval to ensure independence of the atmospheric state for each of the initial conditions.

The departure from the time mean of the $\gamma=2$ zonalmean zonal wind for each of these integrations as a function of latitude and time is shown for representative stratospheric and tropospheric levels in Fig. 5. Considering the upper panels in each frame, it is clear that the stratospheric response begins immediately and is for the most part equilibrated within 50 days. This is not surprising, given the thermal damping time scale of 40 days in the model stratosphere. ${ }^{2}$ Considering the lower panels, the tropospheric response is, on average, established within 200-300 days, but is highly variable for each of

\footnotetext{
${ }^{2}$ This value is taken from Held and Suarez (1994)
}

the members. For example, for the case beginning at day 8001 , the response is not established until day 8700 .

It is also useful to draw attention to the intermittency of the tropospheric response. In some cases, the tropospheric response disappears for a period of a few hundred days and then reappears. This can be seen, for example, near day 7300 of the integration that starts on day 6001 (ensemble member 6 in Fig. 5); we have indicated several of these events with thin dashed vertical lines in the figure. Such tropospheric events are almost always connected to stratospheric warmings, during which the stratospheric jet weakens substantially. Note how the stratospheric disruption precedes the tropospheric one, in these cases, with a time scale much shorter that the adjustment time of several hundred days mentioned above. These disruption events are, we believe, similar to those recently discussed by Baldwin and Dunkerton $(1999,2001)$.

To estimate the average time scales of the response to thermal perturbations, we calculate a scaled measure of the ensemble-mean response. We denote the ensemble- and zonal-mean zonal wind in the transient perturbation experiments $u_{\text {pert }}(k, t)$ for each model level $k$. Consider the departure of the ensemble mean of this quantity from $u(\gamma=2, k)$, which denotes the time- and zonal-mean wind in $\gamma=2$ at each level $k$. We compare this departure to the difference between the mean wind in $\gamma=4$ and $\gamma=2, u(\gamma=4, k)-u(\gamma=2, k)$, since these switch-on experiments should reach the $\gamma=4$ state given enough time. This leads to the following measure of the response at each height:

$$
\Delta(k, t) \equiv \frac{\left\|u_{\text {ert }}(k, t)-u(\gamma=2, k)\right\|}{\|u(\gamma=4, k)-u(\gamma=2, k)\|}
$$




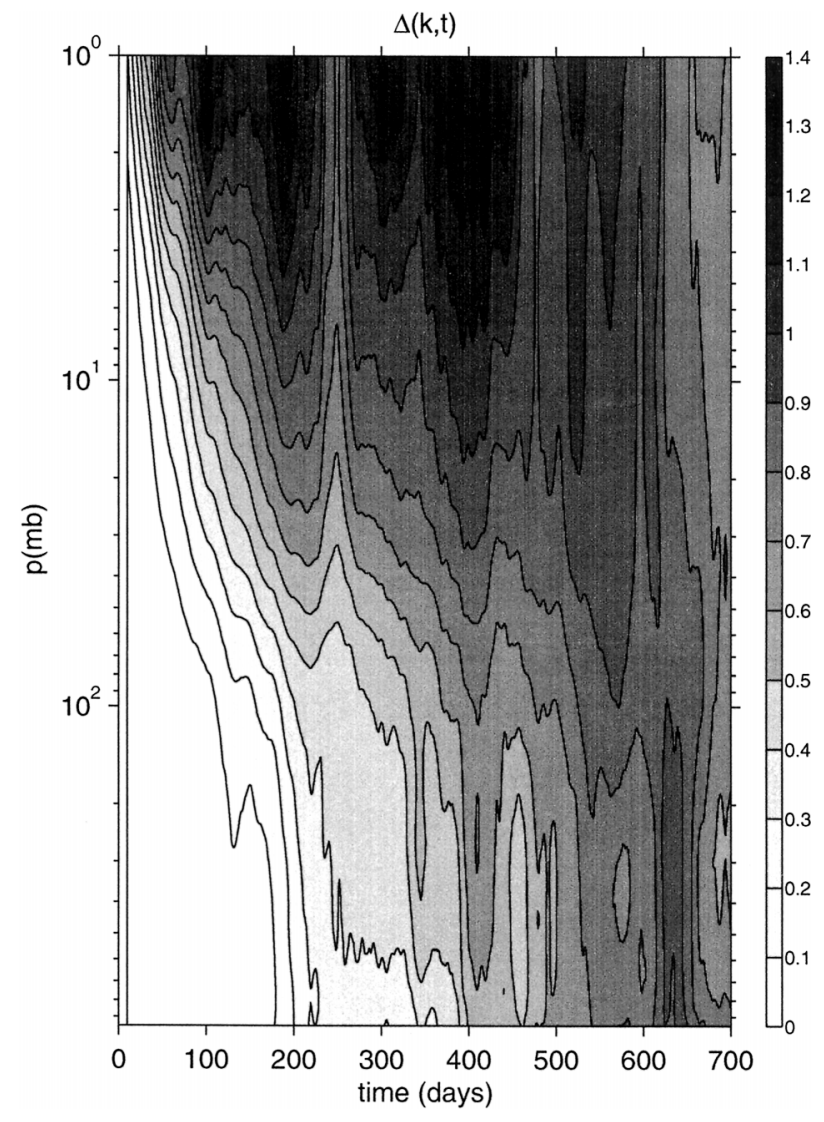

FIG. 6. Plot of $\Delta(k, t)$, defined in (8). The contour and shading interval is 0.1 . A 20-day running mean has been applied to the data.

where $t=0$ at the time the cooling is switched on, and $\|A\|$ is the $20^{\circ}-90^{\circ} \mathrm{S}$ meridional average of the square of the quantity $A$. From this definition, $\Delta(k, t=0)=$ 0 and the low-pass filtered average of $\Delta$ approaches unity as $t \rightarrow \infty$, that is, as the perturbation integration equilibrates to the $\gamma=4$ state. This quantity is plotted, for the first 700 days of the experiments, smoothed with a 20-day running mean, in Fig. 6.

The figure shows that the response descends downward from the upper to the lower stratosphere and into the troposphere. The adjustment occurs in two phases: a linear phase prior to about day 200, and a nonlinear phase after day 200. In the first phase, prior to about day 200, the signal propagates downward to the surface coherently and reaches about $20 \%-30 \%$ of its equilibrium value of unity. During this phase, the phase speed of descent of the signal can be estimated theoretically from the classic work of Dickinson (1968), which is developed further in Haynes et al. (1991). The dynamical problem is that of a transient linear response to switch-on forcing of a thermally damped, zonal-mean flow in a compressible, hydrostatic, rotating, stratified atmosphere. The response to switch-on forcing propagates vertically with phase speed of $C k_{T} H$, where $k_{T}$ is the thermal damping coefficient with value $1 / 40$ day $^{-1}$ in the stratosphere, $H$ is the scale height, and $C$ is an order 1 coefficient. The quantity $k_{T} H$ is equal to 0.19 $\mathrm{km} \mathrm{day}^{-1}$ for a scale height of $7.5 \mathrm{~km}$. From the figure, we can estimate that the signal propagates from 10 to $1000 \mathrm{mb}$ in the range of 100-200 days, which yields an estimated phase speed in the range of $0.17-0.34 \mathrm{~km}$ day $^{-1}$, assuming that the height can be expressed in log pressure coordinates with the same scale height. Thus, the simulated descent of the signal agrees with the theoretical scaling. We have not pursued this analysis to, for example, see if there is a quantitative agreement of the other fields with the transient solutions of Dickinson (1968) and Haynes et al. (1991). However, this analysis suggests that the troposphere is adjusting to a stratospherically induced signal via a "downward control" response, at least in this initial phase, and that aspects of this response may be understood from a linear-theoretic analysis. ${ }^{3}$

In the second phase, subsequent to day 200, the evolution of $\Delta(k, t)$ is noisier, indicating more of a spread between the different ensemble members and a nonlinear (that is, a state-dependent) evolution of the response. During this phase, there is evidence of occasional decreases of $\Delta(k, t)$, for example at day 250 in the stratosphere, that represent reversions of the flow back to the $\gamma=2$ state, but we do not necessarily expect such reversals to be robust to an increase in the number of realizations. This phase of the adjustment occurs more slowly, so that the tropospheric response cannot be said to have equilibrated before day 500 .

The long tropospheric adjustment time scales to external perturbations seen in this simple model are not necessarily surprising, given that internally generated "ultralow-frequency variability" on biennial and even decadal time scales in this kind of simple perpetualsolstice AGCM have been previously reported (James and James 1989; Garric et al. 2003). We are currently investigating whether this aspect of the response is robust to the introduction of a seasonal cycle and to other parameter variations.

\section{Downward-control experiments}

To further explore the idea that the troposphere adjusts to the stratospheric perturbation in a manner consistent with downward-control theory, we present an analysis of a zonally symmetric version of the model. First, consider the $\beta$-plane quasigeostrophic (QG) transformed Eulerian mean (TEM) zonal momentum and thermodynamic equations in log pressure coordinates (Andrews et al. 1987):

\footnotetext{
${ }^{3}$ Alternatively, the rate of descent of the phase of the response could be controlled by eddy mean-flow interaction, in which both the eddy driving and the mean flow are modified (R. A. Plumb 2002, personal communication). We know of no simple way of estimating the phase speed of descent of the response in this case.
} 

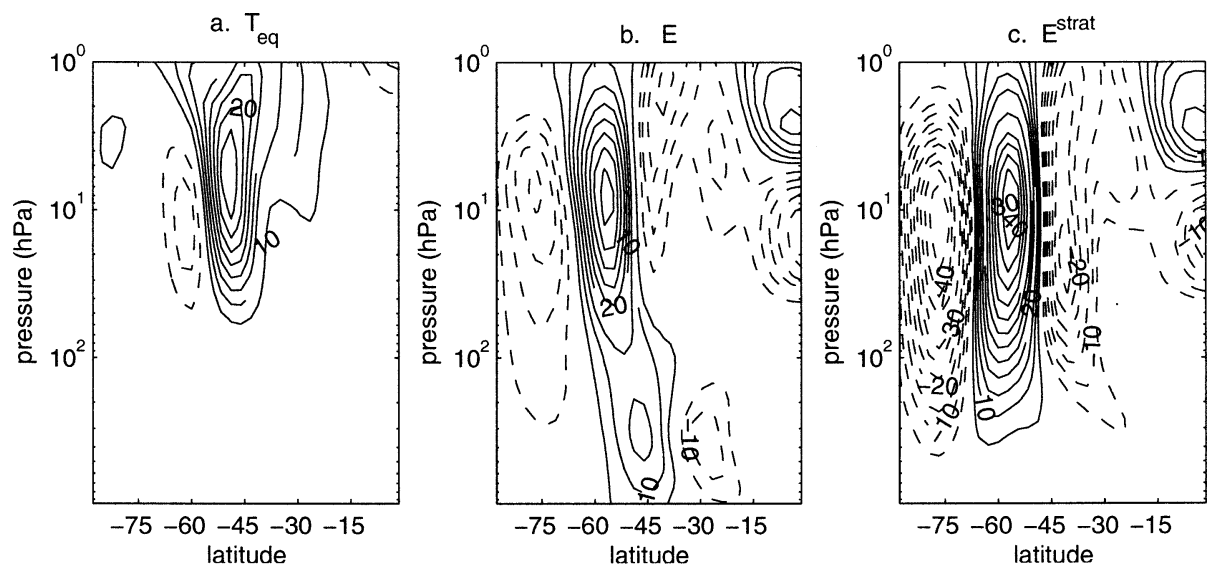

FIG. 7. (a) The quantity $u\left[E(\gamma=2), T_{\mathrm{eq}}(\gamma=4)\right]-u\left[E(\gamma=2), T_{\mathrm{eq}}(\gamma=2)\right]$ described in the text, from the zonally symmetric model. The contour interval is $5 \mathrm{~m} \mathrm{~s}^{-1}$ and the zero contour is not plotted. (b) As in (a) but for $u\left[E(\gamma=4), T_{\text {eq }}(\gamma=2)\right]-u\left[E(\gamma=2), T_{\text {eq }}(\gamma=2)\right]$. (c) As in (b) but for the eddy forcing perturbation confined to the stratosphere, $E^{\text {strat }}[$ Eq. (11)].

$$
\begin{aligned}
\frac{\partial \bar{u}}{\partial t}-f_{0} \bar{v}^{*} & =\frac{1}{\rho_{0}} \boldsymbol{\nabla} \cdot \mathbf{F} \\
\frac{\partial \bar{\theta}}{\partial t}+\theta_{0 z} \bar{w}^{*} & =-k_{T}\left(\theta-\theta_{\mathrm{eq}}\right) .
\end{aligned}
$$

In these equations, $k_{T}$ is the thermal damping coefficient, $\theta_{\mathrm{eq}}$ is the equilibrium potential temperature, and the remaining notation is that of Andrews et al. (1987). From $\gamma=2$ to $\gamma=4$, the eddy forcing $(\boldsymbol{\nabla} \cdot \mathbf{F})$ and the diabatic heating $\left[-k_{T}\left(\theta-\theta_{\mathrm{eq}}\right)\right]$ change simultaneously. But is it the change to the eddy forcing or to the thermal forcing that is responsible for the tropospheric response? Although standard downward-control arguments suggest that only the change in eddy forcing can yield a downward equilibrium response, one would like to know how deep this response is and what shape it takes.

Instead of solving the TEM equations (9)-(10), or their primitive equations generalization (Andrews et al. 1987), we set up a zonally symmetric version of our AGCM. This is a straightforward procedure that is directly related to our model numerics. The output of this model are zonally symmetric winds and temperatures that are equivalent to those obtained from a TEM calculation. The procedure and the validation of the model are outlined in the appendix.

The zonally symmetric model is forced by $T_{\text {eq }}$ given in (1)-(3) and by time- and zonal-mean eddy tendency terms, which we denote $E$ and which are extracted from the eddying AGCM (see the appendix). The zonally symmetric model framework allows us to vary $T_{\mathrm{eq}}$ and $E$ independently, and so to determine the zonally symmetric state of the model that results from these forcings, in the absence of eddy feedbacks. Two inputs control the solutions of the zonally symmetric model: the eddy forcing, which we denote $E$, and the equilibrium temperature $T_{\mathrm{eq}}$. We use the notation $u\left(E, T_{\mathrm{eq}}\right)$ to indicate the solution in the winds $(u)$ that results from a particular eddy forcing $E$ and equilibrium temperature profile $T_{\text {eq }}$. Thus $u\left[E(\gamma=2), T_{\text {eq }}(\gamma=2)\right]$ is the zonal-mean solution when the eddy forcing and the equilibrium temperature are both taken from the $\gamma=2$ case; the zonally symmetric model solution for this case is very nearly identical to the zonal-mean zonal wind shown in Fig. 2a (see appendix).

Figure 7a shows the difference $u\left[E(\gamma=2), T_{\text {eq }}(\gamma=\right.$ $4)]-u\left[E(\gamma=2), T_{\mathrm{eq}}(\gamma=2)\right]$, that is, the change in zonal-mean winds associated with the change in the equilibrium temperature in the absence of eddy feedbacks, with fixed $E(\gamma=2)$. Notice that an eddy-driven circulation is still represented in this solution, but that it is held fixed at the $\gamma=2$ value. The response is confined to the stratosphere. This is expected from standard downward-control arguments, which state that without a change in eddy forcing [for the QG-scaled TEM equations, a change in $\boldsymbol{\nabla} \cdot \mathbf{F}$ in (9)] there can be no induced residual circulation response [a change in $\left.\left(\bar{v}^{*}, \bar{w}^{*}\right)\right]$, and hence the temperature response must be entirely local.

Figure $7 \mathrm{~b}$ shows the difference $u\left[E(\gamma=4), T_{\text {eq }}(\gamma=\right.$ $2)]-u\left[E(\gamma=2), T_{\text {eq }}(\gamma=2)\right]$, that is, the change in zonal-mean winds associated with the change in the eddy forcing from $\gamma=2$ to $\gamma=4$, with fixed $T_{\text {eq }}(\gamma$ $=2$ ). The response extends into the troposphere and in that region strongly resembles the difference between the two cases for the eddying AGCM (Fig. 2c). This result is also expected: because the equilibrium temperature change is zero in any case in the troposphere (Fig. 1c) and because the impact of the equilibrium temperature change in the stratosphere is confined there (Fig. 7a), the tropospheric eddy forcing locally controls the zonal-mean zonal winds in this experiment.

A more interesting experiment would consist in limiting the eddy forcing to the stratosphere alone, and computing the resulting response to determine if and 
how it affects the troposphere below. This is presented in Fig. 7c. To generate this figure, we use $T_{\mathrm{eq}}(\gamma=2)$, as in Fig. 7b, but instead of using $E(\gamma=4)$ everywhere, as in Fig. 7, we transition from $E(\gamma=2)$ in the troposphere $\left(p \geq p_{\mathrm{T}}=100 \mathrm{mb}\right)$ to $E(\gamma=4)$ in the stratosphere $\left(p<p_{\mathrm{T}}=100 \mathrm{mb}\right)$. The eddy forcing profile used is

$$
E^{\text {strat }}=g(p) E(\gamma=2)+[1-g(p)] E(\gamma=4),
$$

where

$$
g(p)=\left\{1+\tanh \left[\log _{10}\left(p / p_{c}\right) / B\right]\right\} / 2
$$

is a weighting function. The parameter $p_{c}$ represents the transition level from $\gamma=2$ below to $\gamma=4$ above, and $B$ determines the sharpness of the transition; in Fig. 7c, we use $p_{c}=100 \mathrm{mb}$ and $B=0.6$. We note in passing that the sharpness of the transition across $p_{c}$ has a somewhat counterintuitive effect on the solution: for smaller $B$, that is, for the $E(\gamma=4)$ forcing more confined to the stratosphere, the wind response penetrates more deeply into the troposphere and the thermal response is strongly localized at $p_{c}$ (not shown). We have chosen a value of $B$ that smooths out this behavior. ${ }^{4}$

Figure $7 \mathrm{c}$ shows that the change to the eddy forcing in the stratosphere does in fact yield a wind response that penetrates well into the troposphere. However, this response only hints at the original tropospheric response pattern in Fig. 2c; in particular, the tropospheric jet does not shift. From this we conclude that, while the change to the eddy forcing in the stratosphere initiates a response in the troposphere, it is insufficient and, without the eddy feedbacks present in the eddying AGCM, cannot yield the full response of the tropospheric circulation.

In order to test this idea, we present the results of two more equilibrated integrations of the eddying AGCM (Fig. 8). In these experiments, we integrate the AGCM with $T_{\text {eq }}(\gamma=2)$ and with an eddy tendency perturbation that is confined to the stratosphere in a manner consistent with the perturbation represented by Fig. 7. That is, we add to the instantaneous tendencies calculated in the model a time-invariant and zonally symmetric tendency perturbation of the form [see (11)(12)]

$$
\begin{aligned}
E^{*} & =E^{\text {strat }}-E(\gamma=2) \\
& =[1-g(p)][E(\gamma=4)-E(\gamma=2)],
\end{aligned}
$$

which is the difference between the $\gamma=2$ and $\gamma=4$ eddy forcing confined to the stratosphere with the

\footnotetext{
${ }^{4}$ The behavior for small $B$ can be understood in terms of the QG residual circulation equations (9), for which the vertical component of the QG EP flux is proportional of the meridional heat flux. As $B$ becomes smaller, this quantity will become more discontinuous at $p_{c}$. Thus, the vertical derivative of this component, which appears as the vertical contribution to $\boldsymbol{\nabla} \cdot \mathbf{F}$ in (9), will be a delta function, which in turn yields a localized residual circulation and temperature response at $p_{c}$.
}
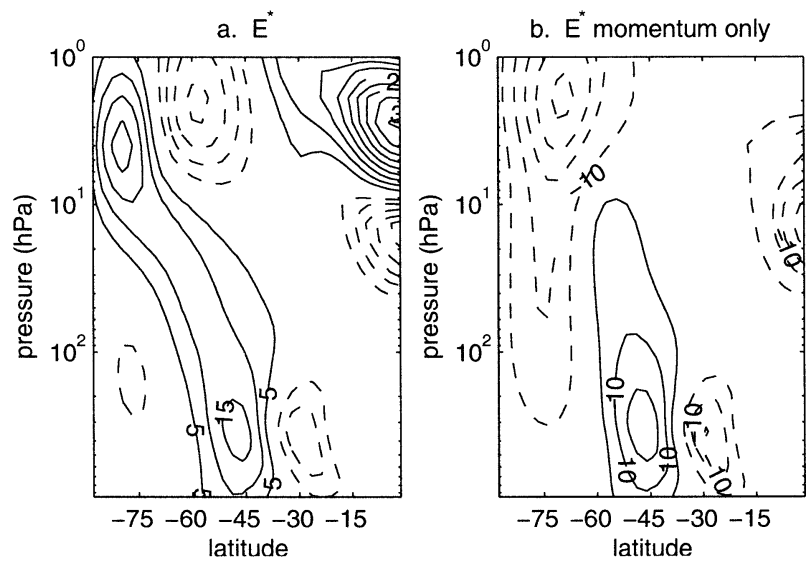

FIG. 8. (a) The zonal-mean zonal wind response from the eddying experiment, with eddy forcing $E^{*}$ [Eq. (13)]. (b) As in (a) but for the zonal-momentum eddy forcing only. The contouring is as in Fig. 7.

weighting function $g(p)$ defined in (12). In the first experiment (Fig. 8a), the eddy forcing perturbation $E^{*}$ is applied to all prognostic-variable tendencies. In the second experiment (Fig. 8b), only the zonal momentum tendency component of $E^{*}$ is used. ${ }^{5}$ The idea of the second experiment is that the vertically integrated eddy momentum flux controls the surface stress, and hence the lower-tropospheric winds, by a vertically integrated momentum balance (W. Robinson and I. Held, 2002, personal communication). The other eddy forcing terms, which, by QG scaling, are dominated by the meridional flux of heat by the eddies and appear as a vertical derivative of a vertical flux, would exert less control.

In Fig. 8, the stratospheric responses are quite distinct from each other and from the original response (Fig. $2 \mathrm{c}$ ); this reflects the strong differences in the stratospheric perturbations. The important point, however, is that the tropospheric, nonlocal, responses in Figs. 2c, $8 \mathrm{a}$, and $8 \mathrm{~b}$ are very similar. Thus, the stratospheric eddy forcing changes and, in particular, the eddy momentum flux changes, diagnosed from the $\gamma=2$ and $\gamma=4$ integrations, are crucial ingredients of the response. The differences in the tropospheric responses between Figs. $7 \mathrm{c}$ and $8 \mathrm{a}$ confirm that the tropospheric eddy feedbacks are also crucial.

We have thus isolated the cause of the tropospheric response to a certain extent, but have not closed the problem because we have not explained what brings about the stratospheric eddy flux change $E^{*}$ itself. Such a closure is difficult because the stratospheric response is highly coupled to the tropospheric response. We illustrate this coupling with a final integration of the zonally symmetric model, which is meant to answer the following question: how does the stratosphere respond (in the absence of eddy feedbacks) to the change in eddy

\footnotetext{
${ }^{5}$ In the spectral model, this is accomplished by determining the vorticity and divergence tendencies that are consistent with a momentum tendency with zero meridional component.
} 


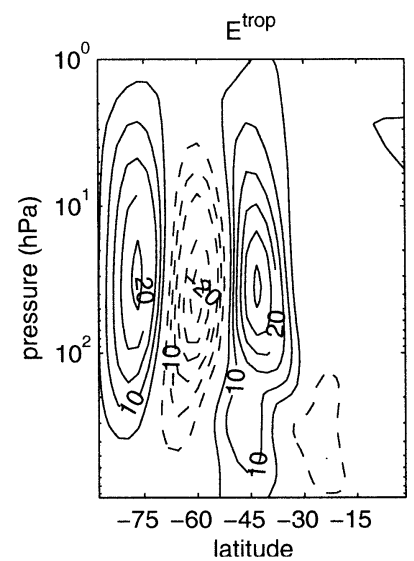

FIG. 9. Zonally symmetric model response to eddy forcing $E^{\text {trop }}$ [Eq. (14)]. The contouring is the same as in Fig. 7.

forcing in the troposphere? One may think of this calculation as the complement of the one present in Fig. 7c. To perform this integration, we use $T_{\text {eq }}(\gamma=2)$ and the following eddy forcing:

$$
E^{\text {trop }}=g(p) E(\gamma=4)+[1-g(p)] E(\gamma=2),
$$

where $g(p)$ is the previous weighting function (12). Thus, we here isolate the eddy forcing perturbation to the troposphere instead of to the stratosphere.

As shown in Fig. 9, the tropospheric response, where the eddy forcing perturbation is applied, is predictably similar to that seen in Figs. 2c and 7b. The fact that this response is weaker is expected from Fig. 7c. What is surprising in Fig. 9, however, is the stratospheric wind response, which is quite barotropic and extends well into the model stratosphere. ${ }^{6}$ This stratospheric response is comparable to, and anticorrelated with, the stratospheric response to the stratospheric eddy forcing in Fig. 7c. This implies that the tropospheric eddy response exerts a great deal of control upon the barotropic wind shear and, presumably, the planetary-wave propagation properties of the stratosphere. This demonstrates that, beyond the prediction that the stratospheric winds will strengthen with increasing $\gamma$, it is difficult to predict the important details of the stratospheric response separately from the tropospheric response.

\section{Conclusions}

In PK, we have shown how our simple model captures key aspects of observed trends in the annular modes in

\footnotetext{
${ }^{6}$ We note that a barotropic wind response above the level of eddy forcing is to be expected from downward-control theory (e.g., Haynes et al. 1991, their Fig. 5). The weak vertical shears in the stratosphere reflects the nonzero value of $g(p)$ above $p_{c}=p_{T}$ and the influence of the sponge in our zonally symmetric model.
}

a robust and reproducible framework. In this study, we have attempted to explain the dynamics of this response. We have found that the equilibrium response in the eddy driving involves a complex combination of changes to the planetary- and synoptic-scale eddy driving; this finding, however, does not constitute an explanation. The transient and zonally symmetric model integrations in sections 4 and 5 provide more insight. Figures 6 and $7 \mathrm{c}$ show that downward control is a crucial ingredient of the response: the stratospheric eddy driving responds in such a way as to "tickle" the troposphere; the troposphere then responds in a more complicated way involving eddy feedbacks. The remaining portion of the response, involving eddy feedbacks, cannot be predicted without a closure theory for the baroclinic eddies. Furthermore, Fig. 9 demonstrates that the stratospheric mean state is itself highly influenced by the change in tropospheric eddy driving that accompanies the poleward shift of the jet. In conclusion, then, from this simple modeling framework the suggestion emerges that stratosphere and troposphere could be coupled in ways that might be rather difficult to untangle.

Acknowledgments. We thank Isaac M. Held, Theodore G. Shepherd, and Walter A. Robinson for comments and suggestions. The work of LMP is supported by a grant from the National Science Foundation.

\section{APPENDIX}

\section{Zonally Symmetric Model}

We illustrate the setup of the zonally symmetric model with an advection equation with a damping term

$$
\frac{\partial S}{\partial t}=-\mathbf{u} \cdot \nabla S-k\left(S-S_{\mathrm{eq}}\right) \equiv F(\mathbf{u}, S)
$$

where $S$ is a tracer; $k$ is a damping rate; and $S_{\text {eq }}$ is a prescribed, time-independent, and longitude-independent equilibrium profile of the tracer. The nonlinear operator $F(\mathbf{u}, S)$ is the instantaneous local tendency of $S$ associated with advection and damping. In our model, analogous operators to $F$ exist for each of the prognostic variables. Consider, now, the time- and zonal mean of (A1), using standard bar and prime notation:

$$
\begin{aligned}
\frac{\partial \bar{S}}{\partial t}=0 \Rightarrow \overline{\mathbf{u}^{\prime} \cdot \nabla S^{\prime}} & =-\overline{\mathbf{u}} \cdot \nabla \bar{S}-k\left(\bar{S}-S_{\mathrm{eq}}\right) \\
& =F(\overline{\mathbf{u}}, \bar{S}) .
\end{aligned}
$$

Thus, if we input the time and zonal-mean state of the model into its tendency operators, we can extract, in a single step, zonal- and time-mean eddy flux tendencies that are exactly consistent with the model numerics.

The zonally symmetric model is integrated with this extracted eddy forcing tendency, $-\mathbf{u}^{\prime} \cdot \nabla S^{\prime}=-F(\overline{\mathbf{u}}, \bar{S})$, 
added to the tendencies solved by the model. For example, illustrating with the tracer equation, we can solve the following:

$$
\begin{aligned}
\frac{\partial \tilde{S}}{\partial t} & =F(\tilde{\mathbf{u}} \tilde{S})-F(\overline{\mathbf{u}}, \bar{S}) \\
& =F(\tilde{\mathbf{u}}, \tilde{S})-\overline{\mathbf{u} \cdot \nabla S^{\prime}} \\
& =-\tilde{\mathbf{u}} \cdot \nabla \tilde{S}-k\left(\tilde{S}-S_{\mathrm{eq}}\right)-\overline{\mathbf{u} \cdot \nabla S^{\prime}}
\end{aligned}
$$

where $\tilde{\mathbf{u}}$ and $\tilde{S}$ are zonal fields that are integrated in time by the model and $F(\tilde{\mathbf{u}}, \tilde{S})$ is the instantaneous zonally symmetric tendency that is calculated by the model. Example (A3) suggests a simple validation test of the model: given the eddy forcing tendency $-F(\overline{\mathbf{u}}, \bar{S})$, if we timestep Eq. (A3) with the zonally symmetric model, we should be able to reproduce the original zonal-mean state of the eddying model. That is, we should obtain $\tilde{\mathbf{u}}=\overline{\mathbf{u}}$ and $\tilde{S}=\bar{S}$ at equilibrium. This will occur provided that the nonlinear zonally symmetric primitive equations are stable and that there exist steady and unique solutions.

We integrate the model for 3000 days and plot fields for a snapshot taken on the last day. According to our testing so far, in the extratropics, the model marches steadily to a unique solution that is independent of initial conditions. We have verified that the zonally symmetric model exactly reproduces the zonal- and time-mean state of the $\gamma=2$ and $\gamma=4$ integrations away from the deep Tropics (not shown). The zonal-mean circulation in the deep Tropics is highly transient and does not settle into an easily sampled equilibrium solution.

The perturbation integrations in section 5 in which the equilibrium temperature is varied correspond, in this example, to keeping the eddy forcing tendency $-\overline{\mathbf{u}^{\prime} \cdot \nabla S^{\prime}}=-F(\overline{\mathbf{u}}, \bar{S})$ fixed while changing the advection/tendency operator $F(\tilde{\mathbf{u}}, \tilde{S})$ to a new operator, $F^{*}(\tilde{\mathbf{u}}, \tilde{S})$. In the tracer equation (A1), this could be accomplished by, for example, varying the equilibrium profile $S_{\text {eq }}$. Conversely, the perturbation integrations in which the eddy forcing is varied while keeping the equilibrium temperature fixed correspond to changing the eddy forcing tendency while keeping the operator $F$ fixed.

\section{REFERENCES}

Andrews, D. G., J. R. Holton, and C. B. Leovy, 1987: Middle Atmosphere Dynamics. Academic Press, 489 pp.

Baldwin, M. P., and T. J. Dunkerton, 1999: Propagation of the Arctic Oscillation from the stratosphere to the troposphere. J. Geophys. Res., 104, 30 937-30946.

— and - 2001: Stratospheric harbingers of anomalous weather regimes. Science, 294, 581-584.

—_, X. Cheng, and T. Dunkerton, 1994: Observed correlations between winter-mean tropospheric and stratospheric circulation anomalies. Geophys. Res. Lett., 21, 1141-1144.

COESA, 1976: U.S. Standard Atmosphere, 1976. U.S. Government Printing Office, $227 \mathrm{pp}$.

Dickinson, R. E., 1968: On the excitation and propagation of zonal winds in an atmosphere with Newtonian cooling. J. Atmos. Sci., 25, 269-279.
Fusco, A., and M. Salby, 1999: Interannual variations of total ozone and their relationship to variations of planetary wave activity. J. Climate, 12, 1619-1629.

Fyfe, J. C., G. J. Boer, and G. M. Flato, 1999: The Arctic and Antarctic Oscillations and their projected changes under global warming. Geophys. Res. Lett., 26, 1601-1604.

Garric, G., S. A. Venegas, C. Tansley, and I. James, 2003: Atmosphere-sea ice low-frequency variability with a simple model of the Southern Hemisphere. Quart. J. Roy. Meteor. Soc., 129, 2347-2366.

Gillett, N. P., M. R. Allen, and K. D. Williams, 2002: The role of stratospheric resolution in simulating the Arctic Oscillation response to greenhouse gases. Geophys. Res. Lett., 29, 1500, doi: 10.1029/2001GL014444.

Haynes, P. H., M. E. McIntyre, T. G. Shepherd, C. J. Marks, and K. P. Shine, 1991: On the "downward control" of extratropical diabatic circulations by eddy-induced mean zonal forces. J. Atmos. Sci., 48, 651-680.

Held, I. M., and M. J. Suarez, 1994: A proposal for the intercomparison of the dynamical cores of atmospheric general circulation models. Bull. Amer. Meteor. Soc., 75, 1825-1830.

$\mathrm{Hu}$, Y., and K. K. Tung, 2002: Interannual and decadal variations of planetary wave activity, stratospheric cooling, and the Northern Hemisphere Annular Mode. J. Climate, 15, 1659-1673.

James, I. N., and P. M. James, 1989: Ultra-low-frequency variability in a simple atmospheric circulation model. Nature, 342, 53-55.

Newman, P. A., E. R. Nash, and J. Rosenfield, 2001: What controls the temperature of the Arctic stratosphere during the spring? $J$. Geophys. Res., 106, 19 999-20 010.

Perlwitz, J., and H.-F. Graf, 1995: The statistical connection between tropospheric and stratospheric circulation of the Northern Hemisphere in winter. J. Climate, 8, 2281-2295.

Plumb, R. A., and K. Semeniuk, 2003: Downward migration of extratropical zonal wind anomalies. J. Geophys. Res., 108, 4223, doi:10.1029/2002JD002773.

Polvani, L. M., and P. Kushner, 2002: Tropospheric response to stratospheric perturbations in a relatively simple general circulation model. Geophys. Res. Lett., 29, 1114, doi:10.1029/ 2001 GL014284.

Scinocca, J. F, and P. Haynes, 1998: Dynamical forcing of stratospheric planetary waves by tropospheric baroclinic eddies. $J$. Atmos. Sci., 55, 2361-2392.

Shindell, D. T., R. L. Miller, G. Schmidt, and L. Pandolfo, 1999: Simulation of recent northern winter climate trends by greenhouse-gas forcing. Nature, 399, 452-455.

—_, G. A. Schmidt, R. L. Miller, and D. Rind, 2001: Northern Hemisphere winter climate response to greenhouse gas, ozone, solar, and volcanic forcing. J. Geophys. Res., 106, 7193-7210.

Simmons, A. J., and D. M. Burridge, 1981: An energy and angularmomentum conserving vertical finite difference scheme and hybrid vertical coordinates. Mon. Wea. Rev., 109, 758-766.

Taguchi, M., and S. Yoden, 2002a: Internal interannual variations of the troposphere-stratosphere coupled system in a simple global circulation model. Part I: Parameter sweep experiment. J. Atmos. Sci., 59, 3021-3036.

- , and $-2002 \mathrm{~b}$ : Internal interannual variations of the troposphere-stratosphere coupled system in a simple global circulation model. Part II: Millennium integrations. J. Atmos. Sci., 59, 3037-3050.

- T. Yamaga, and S. Yoden, 2001: Interannual variability in the troposphere-stratosphere coupled system simulated in a simple global circulation model. J. Atmos. Sci., 58, 3184-3203.

Thompson, D. W. J., and J. M. Wallace, 1998: The Arctic Oscillation signature in the wintertime geopotential height and temperature fields. Geophys. Res. Lett., 25, 1297-1300.

_, and — - 2000: Annular modes in the extratropical circulation. Part I: Month-to-month variability. J. Climate, 13, 1000-1016. , and S. Solomon, 2002: Interpretation of recent Southern Hemisphere climate change. Science, 296, 895-899.

, J. M. Wallace, and G. C. Hegerl, 2000: Annular modes in the extratropical circulation. Part II: Trends. J. Climate, 13, 10181036. 